\section{LA DEFENSA, POLÍTICA DE ESTADO}

\author{
Alejandro Muñoz-Alonso \\ Senador por Madrid \\ Portavoz del Grupo P. Popular en la Comisión \\ de Defensa del Senado (2004-2008)
}

DEFENSE, AS A STATE'S POLICY
ABSTRACT: The areas of the State Policy are set, specially focussing on the Defense as it is closely related to concepts like National Interest and Anglo-Saxon bipartisan policy. Nevertheless, the political alternation will cause differences. It is also necessary to tell the difference between the State Policy and the Policy carried out by the State. That's why the democratic Parliaments play a fundamental role in shaping the defence policy. Although the Parliament symbolizes the National Sovereignty there can be and there are actually differences with the Public Opinion. This sort of divorce is not really new as, in Spain, it became evident in a very intensive way on the occasion of decisions such as the Spanish entry into the NATO. A State Policy related to Defense, kept during a long time and resistant to political alternation would certainly be the best instrument to reach a culture of defense.

KEY WORDS: State policies; bipartisan policy; democratic parliaments; defense culture.

\section{Los ámbitos de las "políticas de Estado"}

La expresión "política de Estado" se utiliza con mucha frecuencia en el lenguaje político habitual -seguramente con excesiva frecuencia- pero normalmente no se define, dando por supuesto que todo el mundo entiende su auténtico significado. Evidentemente con esa expresión se quiere decir que, si bien en un régimen democrático de alternancia en el poder lo normal es que las políticas que aplican los diferentes gobiernos cambien de orientación al sucederse en las responsabilidades del poder equipos, partidos políticos o coaliciones con distintos programas de actuación política, en ciertos ámbitos especialmente vinculados con la propia identidad del Estado, con sus instituciones básicas, con sus procedimientos fundamentales de actuación, con su proyección hacia el exterior y sus relaciones con otros sujetos de Derecho Internacional y con
RESUMEN: Se establecen los ámbitos de las políticas de estado, con especial énfasis en la Defensa al estar esta vinculada al concepto de interés nacional, y al anglosajón bipartisan policy. No obstante, la alternancia política va a crear discrepancias. Conviene diferenciar la "Política de Estado" y la "Política del Estado". Por ello, los Parlamentos democráticos juegan un papel fundamental en la conformación de la política de defensa. Pese a que el Parlamento representa a la soberanía nacional pueden producirse, y de hecho se producen, discrepancias con la opinión pública. Este divorcio no es nuevo y en España se ha manifestado de forma muy intensa con ocasión de tomas de decisión como la incorporación a la OTAN. Una política de Estado en defensa, mantenida en el tiempo y resistente a las alternancias políticas sería el instrumento más adecuado para lograr una cultura de defensa.

PALABRAS CLAVE: Políticas de estado; bipatisan policy; parlamentos democráticos; cultura de defensa.

su necesaria permanencia y continuidad, es conveniente -e incluso necesario- que las políticas, al menos en sus grandes líneas maestras, no cambien sino que se sucedan en una continuidad identificable.

Eso significa que se trata de ámbitos donde las políticas deben ser decididas -o es conveniente que lo sean- por un amplio consenso de los principales partidos que compongan el arco parlamentario. Idealmente por todos pero, dado que esa unanimidad es siempre o casi siempre muy difícil de alcanzar, al menos por los partidos más representativos. La fórmula mínima sería que en ese consenso participaran el partido o los partidos que forman el gobierno $y$, al menos, el más representativo de los partidos de la oposición. Aunque si en la oposición existen dos o más partidos que tienen similar peso político parece lógico que no se debería prescindir de ninguno de ellos. 
Este concepto de "política de Estado", cuya relevancia práctica difícilmente se puede exagerar, no tiene sin embargo una especial consideración constitucional. Las Constituciones no definen cuáles deban ser consideradas como "políticas de Estado". Sí establecen para la aprobación de leyes relativas a materias de especial importancia requisitos procedimentales especiales, como hace la Constitución española de 1978 (CE), en relación con las leyes orgánicas. Pero no sería adecuado confundir o identificar las materias que deben ser objeto de ley orgánica con las políticas de Estado, aunque el requisito de la mayoría absoluta exigido para la aprobación de las leyes orgánicas parezca estar ya apuntando en esa dirección. Aunque no sea conveniente confundir un procedimiento especial de elaboración de una ley, como el que se exige para las leyes orgánicas, con la relevancia política que se atribuye a las políticas de Estado, no cabe duda de que hay puntos de contacto entre esos diversos niveles. Por lo pronto, en opinión de quien esto escribe, no habría ningún inconveniente en considerar que todas las materias que el artículo 81 de la CE considera especificamente como propias de ley orgánica (desarrollo de los derechos fundamentales y de las libertades públicas, las que aprueben los Estatutos de Autonomía y el régimen electoral general) constituyen "políticas de Estado" en el sentido en que hemos definido este concepto. Por poner un ejemplo concreto, es una idea generalmente admitida que un determinado gobierno de un partido, aun contando con mayoría absoluta en el Parlamento, no debería afrontar un cambio de la ley electoral sin contar con el consenso, como mínimo, del principal partido de la oposición. En otro caso la nueva ley electoral, cuya "legalidad" nadie podría negar, carecería de "legitimidad" y tendría escasa duración en el tiempo. Tal fue el caso, por ejemplo, de la reforma electoral realizada por Mitterrand en 1985. Este ejemplo de la ley electoral es oportuno porque se suele estimar que los procedimientos electorales constituyen "las reglas de juego" que ni pueden ni deben alterarse sin el concurso de quienes participan en ese "juego" que es una contienda electoral.

Pero, evidentemente, ahí no quedaría cerrado el catálogo de las políticas de Estado que, según la mayor parte de los especialistas y de los propios dirigentes políticos, debería incluir otros ámbitos de especial importancia, según veremos a continuación, como la política exterior, la seguridad y la defensa y la lucha contra el terrorismo. En sentido contrario, se percibe a veces una tendencia a ampliar excesivamente el ámbito de estas políticas de Estado a terrenos que, sin mengua de su importancia intrínseca, no exigirían ese especial tratamiento. Por poner algunos ejemplos a los que en tiempos recientes se ha etiquetado en España -en nuestra opinión abusivamente- como políticas de Estado, no deberian tener ese carácter las normas relativas a la discapacidad o a la llamada violencia de género por relevantes que sean en un determinado momento político. Y lo mismo podríamos decir de otros sectores de actuación gubernamental como la vivienda o las políticas activas de empleo. Un caso especial sería la normativa relativa a la seguridad social que, al exigir una continuidad que garantice el futuro de las pensiones, en España ha sido objeto de una política de Estado que tuvo su concreción en el Ilamado Pacto de Toledo.

En las políticas de Estado, así definidas, es necesario, en todo caso, distinguir varios niveles. Si partimos del supuesto de que, sobre todo a los efectos de este artículo, es en los ámbitos de la política exterior y de la defensa donde las políticas de Estado tienen una razón de ser más sólida y comprensible, sería conveniente señalar que en ningún caso sería necesario que en todas y cada una de las acciones que se lleven a cabo por un determinado gobierno en esos ámbitos de actuación política se lograra ese amplio consenso. Una política de Estado, basada en un consenso tan amplio como fuera posible, sería necesaria, o al menos conveniente, en lo que respecta a la legislación básica sobre estas materias, tratados internacionales de especial importancia, adhesión o integración en organizaciones internacionales..., etc. La organización básica del servicio exterior, la determinación del modelo de Fuerzas Armadas o la organización básica de la defensa y la seguridad nacionales... serían algunos ejemplos de políticas de Estado, al menos deseablemente. Pero no sería prudente considerar como tales las actuaciones concretas, sin carácter legislativo que un gobierno toma a diario sobre estas materias.

En estos tiempos posteriores a la Guerra Fría en los que se ha ido perfilando como una nueva amenaza el terrorismo, tanto el de raíz nacionalista -en España sería el caso de ETA- como el nuevo terrorismo Ilamado internacional, esencialmente de raíz islamista, es también un valor entendido que la lucha contra el terrorismo debería incluirse entre las políticas de Estado. A ese enfoque ha respondido 
en España, mientras duró, el Pacto por las Libertades y contra el Terrorismo.

\section{Defensa e interés nacional}

La idea de que la política de defensa, como la política exterior, son "políticas de Estado" es una afirmación que se repite insistentemente, casi como un mantra que se salmodia una y otra vez, como si se intentase conjurar la aparición de discrepancias en este importante reducto, tan ligado a la propia identidad y la definición que de sí mismo da cualquier sistema político. Mientras que en cualquier otro ámbito de acción política se considera, en una sociedad democrática, que lo normal es la diversidad de opciones y opiniones, que se resuelven por el democrático procedimiento de la mayoría, cuando se trata de la política de defensa, como ocurre con la política exterior -tan ligadas entre sí, por otra parte- se suele partir del supuesto de que lo deseable es el consenso, al menos entre los grupos o partidos más significativos.

En mi opinión, esto se debe a la estrecha conexión que se supone que existe entre estas políticas y el concepto de interés nacional que, como explicó Charles A. Beard (The Idea of National Interest, 1934), comienza a configurarse en el siglo XVIII y, paulatinamente, desplaza a otros criterios de acción política como "la voluntad del príncipe" - la raison d'etat que habían imperado hasta aquel momento. Frente a la caprichosa voluntad del monarca que se impone incluso por encima de la ley, el concepto de interés nacional implica una cierta objetivación, al menos aparente, de las motivaciones del gobernante, sobre todo en el ámbito de la acción exterior y militar. Es más, los Estados del continente europeo, contiguos entre sí, dan a estas primeras formulaciones del interés nacional un contenido fundamentalmente militar, en el marco de una preocupación prioritaria por la seguridad. Territorios y fronteras son el contenido esencial de lo que entonces se entendía por interés nacional. El sistema de Westfalia parte del supuesto de que todos los Estados son soberanos $y$, por lo tanto, iguales entre sí, pero en la realidad son evidentes las diferencias: Hay Estados expansivos con fuertes tendencias a la hegemonía, regional, continental o incluso extracontinental que deben ser contenidas 0 frenadas por medio de coaliciones que intentan establecer unos mecanismos de seguridad. Esta idea se convierte, ya desde entonces, en un componente esencial de las políticas de defensa. Precisamente, Inglaterra hará de la balance of power, del equilibrio entre las potencias continentales, el eje de su política exterior e identificará con ese concepto su propia idea del interés nacional.

Además, la posición geopolítica de Inglaterra y su estructura económica, en la que el comercio exterior ocupaba un lugar preferente, daban allí a la idea del interés nacional un contenido diferente al de otros países: En la visión inglesa, los aspectos comerciales y sus derivados, como el principio de la libertad de los mares, adquirian un carácter prioritario. Se explica así la tradición británica de un poder naval de primer nivel, así como la inexistencia de un ejército de tierra permanente $y$, en todo caso, de dimensiones muy reducidas.

Esta concepción del interés nacional alcanzará su madurez en el siglo XIX y será Palmerston quien hará su formulación clásica y más conocida. Tras la victoria en la guerra de Crimea y cuando Gran Bretaña es la indiscutible potencia hegemónica, no sólo en Europa sino en el mundo, el primer ministro británico (1856) no dejará lugar a dudas acerca de cuál es el criterio de toda su acción exterior: "Hacer de los intereses de nuestro país el único principio director". "Inglaterra no tiene aliados eternos ni enemigos permanentes. Sólo son eternos nuestros intereses y nuestro deber de defenderlos". De ahí saldrán la poca justificada idea del "espléndido aislamiento" británico o la imagen de la "Pérfida Albión", expresión de las críticas, de la "leyenda negra" de que siempre son objeto las potencias hegemónicas o imperiales.

Pero la identificación de defensa e interés nacional tampoco resuelve definitivamente el problema porque este último concepto está muy lejos de ser una evidencia. Precisamente una de las claves más importantes que explican las diferencias entre los diversos partidos políticos radica en las distintas visiones que tiene éstos del interés nacional. El problema se complica si consideramos que se suele distinguir entre intereses vitales e intereses secundarios y hay un acuerdo entre los expertos en el sentido de que el primero y más importante de los intereses vitales es la supervivencia del Estado que, en cualquier caso y situación exige el uso y aplicación de todos los recursos diplomáticos y de cualquier otra clase de que disponga el Estado 
incluido, si fuera necesario, el uso de la fuerza militar. Resulta difícil imaginar una situación en la que estando en juego la supervivencia del Estado no se lograra alcanzar el consenso entre todas las fuerzas políticas para garantizar esa supervivencia. Pero, ¿qué ocurre si, por azares de la política, contribuyen a la formación de la voluntad del Estado grupos separatistas cuyo objetivo último es, precisamente, debilitar y aniquilar al Estado? Sería preciso introducir aquí un concepto elaborado en Alemania como criterio para las relaciones entre el Gobierno federal y los Länder: nos referimos al concepto de lealtad institucional que, si falta, hace imposible cualquier debate sobre el interés nacional o sobre una supuesta política de Estado. Algunas de las vicisitudes que se han vivido en España en estos últimos años nos demuestran, sin lugar a dudas, que no estamos refiriéndonos a hipótesis de escuela sino a situaciones que se pueden dar $y$, de hecho, se dan, por extraño que pueda parecer ${ }^{1}$.

Por otra parte, el concepto de interés nacional sufre actualmente una crisis patente ya que se le asocia con las doctrinas del realismo político y del llamado hard power, propicio a la solución militar de los conflictos internacionales y basado, según esos críticos, en una visión egoísta de las relaciones internacionales. Los "políticamente correctos" defensores del soft power, del diálogo sin límites, del multilateralismo y del apaciguamiento a toda costa no aceptan el interés nacional como criterio para determinar las políticas exteriores y de defensa, ya que lo asocian con un supuesto unilateralismo, basado en una visión egoísta y "nacionalista" de las relaciones internacionales. El interés nacional estaría para quienes así piensan en los antípodas de una concepción idealizada y utopizante de las relaciones internacionales, propia de ciertos sectores de la izquierda, que recientemente ha sido denominada "buenismo"2.

\section{Coincidencias y discrepancias en la política de DEFENSA}

Para intentar profundizar un poco más en la idea de la política de defensa como política de Estado sería conveniente que analizáramos, al menos someramente, cómo se afronta esta cuestión en alguno de los países más destacados e influyentes.
A lo largo del siglo $X X$, no son pocos los análisis que han considerado a la bipartisan policy como un rasgo característico de la política de los Estados Unidos en política exterior y defensa, de modo que, en definitiva y según este enfoque, demócratas y republicanos acabarían siempre adoptando las mismas políticas ante problemas o situaciones similares. Pero esta afirmación -que pudo tener una cierta justificación o realidad, con muchas reservas, durante el largo período de la Guerra Fría- no resiste un análisis detenido. Tanto en el terreno de las teorías sobre la política exterior y de defensa, como en el de las políticas realmente aplicadas, en los Estados Unidos son perfectamente detectables las diferencias que han existido y que existen, en este ámbito, entre los demócratas y los republicanos, diferencias que, por otra parte, no siguen en absoluto la divisoria entre estos dos grandes partidos.

En el terreno de la teoría, se describen escuelas diferentes e incluso antitéticas de política exterior y defensa en aquel pais, que marcan con su sello diverso, en uno $u$ otro momento, las decisiones y actuaciones concretas de las presidencias. Cada uno de estos enfoques trata, lógicamente, de imponerse sobre los demás y convertirse así en la bipartisan policy, pero la realidad nos enseña que casi nunca se logra este supuesto consenso. Más allá de las distintas escuelas -hamiltonianos, wilsonianos, jeffersonianos y jacksonianos- que distingue Walter Russell Mead $^{3}$ o del esquema más simple de idealistas y realistas, que, a partir de Henry Kissinger ${ }^{4}$, han elaborado más recientemente Pierre Hassner y Justin Vaïse ${ }^{5}$, es evidente que en los Estados Unidos no sólo existen diversas escuelas de pensamiento, sino también políticas diferentes, desde un tradicional aislacionismo a diversas posiciones acerca de la implicación americana en los asuntos del mundo: unilatelarismo, internacionalismo, multilateralismo..., etc., que siendo en principio enfoques diversos de política exterior tienen inmediatas repercusiones en el ámbito de la defensa. Ante ese maremágnum de escuelas y tendencias resulta poco oportuno hablar de bipartisan policy, de consenso o de política de Estado.

Por referirnos exclusivamente a la más reciente de las crisis vividas por los Estados Unidos en el ámbito de la política exterior y de defensa, es evidente que el presidente Geoge W. Bush, tras lograr un apoyo casi unánime en ambas Cámaras del Congreso para iniciar la intervención en Iraq, ha visto cómo se multiplicaban las críticas procedentes de 
sectores muy diversos del arco político y de la sociedad, incluidos algunos relevantes personajes de su propio partido. $Y$ estas discrepancias sobre la intervención en Iraq no han sido una excepción sino que, más bien, constituyen la regla general de la política norteamericana. Bastaría recordar las resistencias, procedentes de los sectores aislacionistas, a las que tuvo que enfrentarse Rooselvet antes de entrar en la Segunda Guerra Mundial. Sólo el ataque de Pearl Harbor y la insensata declaración de guerra por parte de Hitler logran hacer realidad la unanimidad nacional y convierten la guerra en una bipartisan policy. Otro ejemplo histórico relevante, que muestra las más agudas discrepancias en el terreno militar y de la defensa es el de la guerra de Vietnam, que por sí solo requeriría un reflexión, pues difícilmente se podría considerar como expresión de una política de Estado, aquel conflicto que dividió tan profundamente a la sociedad norteamericana.

También hay que tener en cuenta que, en una coyuntura electoral, la coincidencia de los contendientes en una misma política puede tener un cierto carácter desmovilizador ya que el elector puede estimar que si, gane uno u otro, se va a aplicar la misma política no vale la pena molestarse en ir a las urnas. Esto podría explicar que, durante la campaña electoral presidencial de 2004, el candidato demócrata, Kerry, tratara de presentar una política respecto de Iraq muy diferenciada de la de Bush, a pesar de que, en su momento, su voto personal, como el de casi todos los demócratas, había sido favorable a la intervención militar contra la dictadura de Sadam Husein. Pero, ¿habría sido su política, de haber ganado, diferente de la de Bush? No han sido pocos los comentaristas que han estimado que una hipotética Administración demócrata no habría podido diferenciarse demasiado de la republicana de Bush. Podría incluso afirmarse que, en ciertos casos, las exigencias de una política de Estado de imponen por sí mismas y que, pese a ciertas promesas electorales, una vez instalados en las responsabilidades del poder, los nuevos gobernantes no pueden a veces diferenciarse demasiado de sus predecesores. Podría plantearse en esta línea y sin salir del problema de Iraq la siguiente cuestión: De haber ganado las elecciones en 2000 el candidato demócrata Gore en vez del republicano Bush, ¿habría habido invasión de Iraq? Evidentemente se pueden imaginar respuestas para todos los gustos pero la hipótesis de que incluso con un demócrata en la Casa Blanca podría haber habido intervención militar en Iraq no puede descartarse si pensamos que la secretaria de Estado del demócrata Clinton, Madeleine Albright, reconoce en sus Memorias cómo aquella Administración adoptó la política de "cambio de régimen" como "objetivo explícito de la política de Estados Unidos" y cómo "la mezcla de sanciones, contención, desafíos iraquíes y nuestra incertidumbre sobre las armas de Sadam no podía continuar indefinidamente" y concluye: "Como dijo el presidente Clinton en 1998, el líder iraquí amenaza la 'seguridad mundial' y la mejor manera de terminar con esa amenaza de una vez y para siempre es con un nuevo gobierno iraquí" 6 . En síntesis, se puede mantener la hipótesis de que, en el caso de Iraq, un supuesto interés nacional de los Estados Unidos -acertado o no, eso es otra cuestión- habría llegado posiblemente a la intervención militar, cualquiera que hubiera sido la Administración gobernante en los Estados Unidos.

Los anteriores casos confirmarían que, de todos modos, hay situaciones en las que, bien por razones de interés nacional compartidas por los grandes actores políticos o por cualesquiera otras circunstancias en las que se impone una determinada "política de Estado" que uno u otro partido político en el poder se ve obligado a ejecutar. Las más evidentes, por supuesto, serían las posibles situaciones excepcionales, de crisis existencial, sobre todo aquellas que ponen en peligro la propia supervivencia del Estado en las que lo normal es que se logre la unanimidad nacional y la defensa del territorio nacional o del propio sistema político se convierte en una "política de Estado", basada en un amplio consenso nacional. Tal sería el caso de las guerras defensivas ante una agresión exterior, especialmente la invasión del territorio nacional por parte de una potencia extranjera. En tales casos, se suelen conseguir los más amplios apoyos en los diversos sectores políticos y de opinión, pero esto no es nunca una verdad absoluta. Se recuerda la famosa union sacrée que alineó a todos los partidos franceses de la derecha a la izquierda contra Alemania en la Primera Guerra Mundial, incluidos los socialistas que habian hecho gala de su antibelicismo. Pero en la Segunda Guerra Mundial y aunque a las historias oficiales no les guste recordarlo, fueron muchos los franceses que aceptaron el régimen colaboracionista del mariscal Petain y, al principio, más bien pocos los que se sumaron a la empresa de de Gaulle que, inicialmente, parecía tan imposible como desesperada.

En el caso británico es bien conocido el agudísimo contraste entre el apaciguamiento de Neville Chamberlain que, con el francés Daladier firmó con Hitler el infausto

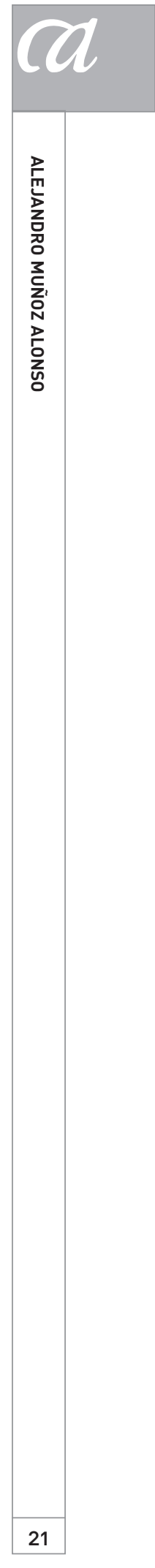

ARBOR CLXXXIV Anejo 2 [2008] 17-29 ISSN: 0210-1963 
acuerdo de Múnich y la posición de Churchill, decidido a resistir a la amenaza nazi, aunque fuera al precio de "sangre, sudor, esfuerzo y lágrimas". Ya en pleno curso de la Segunda Guerra Mundial se perfilan posiciones diferentes entre quienes apuestan por la rendición incondicional de la Alemania nazi y los que aspiraban a un armisticio, que habría supuesto una negociación con Hitler.

Todos estos ejemplos históricos muestran que, en cualquier caso, las diferencias en estos ámbitos de la política exterior y de defensa se dan también, por lo menos si no más, como en cualquier otro terreno. Sólo en casos muy excepcionales de gran crisis nacional y casi siempre de un modo fugaz, estas políticas, apoyadas por los principales partidos y por amplios sectores de la opinión pública se convierten en verdaderas "políticas de Estado", fruto de un extenso consenso nacional.

En los períodos de paz es mucho más difícil lograr ese amplio apoyo que es el más sólido fundamento de las "políticas de Estado". Quizás el caso más peculiar de política de Estado en el ámbito de la defensa es el que representa Francia donde el gaullismo, tal y como lo concibió el general De Gaulle ha impreso su huella indeleble en la política de aquel país. No hubo enemigo más declarado de las aspiraciones nucleares del general que Mitterrand, que, con contundencia, se negó a avalar la creación de la force de frappe nuclear para, una vez que llegó a la presidencia de la República, convertirse en su más decidido adalid. $Y$ algo parecido podríamos decir de la posición francesa respecto de los Estados Unidos, teñida de un antiamericanismo indisimulable y compartida allí con algunos matices tanto por la derecha como por la izquierda. Aunque desde la llegado al Elíseo de Sarkozy parece perfilarse una nueva actitud hacia los Estados Unidos y hacia la OTAN. Pero incluso en este caso francés, un análisis más detenido nos lleva necesariamente a la conclusión de que tanto en la época gaullista ${ }^{7}$ como durante la presidencia de Chirac, tras la fachada de una casi unanimidad nacional persistían diferentes enfoques, como, por otra parte, es natural en una sociedad pluralista. Algo parecido podría decirse de la actual presidencia de Sarkozy y de su decisión de volver, tras más de cuarenta años, a la estructura militar de la OTAN, discutida incluso por miembros relevantes de su propio partido.

En España hemos presenciado cómo la llegada al poder de PSOE en 2004 ha supuesto un vuelco tanto en el terreno de la política exterior como en la defensa, aunque desde el punto de vista de los socialistas habría sido el anterior gobierno del Partido Popular el que se habría alejado de una supuesta política tradicional en estos ámbitos. En nuestra opinión este último enfoque no estaría justificado, pero aun admitiendo que se trata de una cuestión discutible, la evidencia es que, en el momento presente, los dos grandes partidos españoles no coinciden ni en su visión de la política exterior ni en algunos de los fundamentos sobre los que deba asentarse la política de defensa, como muestra, en este último caso, el voto del Partido Popular contrario a la Ley Orgánica de la Defensa Nacional, aprobada por las Cortes Generales en 2005. Quizás nos hallamos ante una especie de vuelta al pasado, a los momentos iniciales de la Transición democrática, cuando las divergencias entre la derecha y la izquierda en estas materias era patente, como puso de relieve la larga polémica en torno a la adhesión y permanencia en la OTAN. La Alianza Atlántica, en la España de aquellas décadas, jugó el papel de contradictorio punto de referencia y línea divisoria entre dos concepciones antitéticas de la política de defensa y de algunos de los aspectos más notables y significativos de la política exterior, como es la cuestión de las relaciones con los Estados Unidos, cargadas, precisamente, desde los acuerdos firmados con este país por Franco en 1953, con un fuerte componente defensivo.

Por otra parte, la cuestión de la OTAN puede también considerarse como un caso en el que, en definitiva, se impone una cierta idea del interés nacional, más allá de las posiciones partidistas, Cuando en 1981-1982 el PSOE batallaba en contra de la permanencia en la OTAN y a favor de un referéndum "para salirse de la OTAN", algunos vaticinamos que, una vez en el poder, el nuevo Gobierno socialista, de una u otra manera, se convertiría al atlantismo y defendería la permanencia en la Alianza. El camino fue largo y difícil y pasó porque el presidente González se desembarazara de su primer ministro de Asuntos Exteriores, Fernando Morán, furibundo antiatlantista. Pero, en definitiva, se impuso lo que bastantes veíamos como patente interés nacional y la permanencia en la OTAN se convirtió en una "política de Estado", defendida por más del $90 \%$ del arco parlamentario. Evidentemente, no todos los atlantistas son iguales, no todos ven o conciben a la OTAN de la misma manera ni todos contemplan del mismo modo la presencia y el papel de España en la organización, pero todos esos matices no obstan al carácter de verdadera "política de Estado" que tiene esa importante opción de 
política exterior y de defensa. Aunque en algunos sectores de la izquierda socialista el "entusiasmo" por la OTAN sea perfectamente descriptible. Frecuentemente hemos oído la expresión "menos OTAN y más ONU", más Ilena de ingenuo utopismo que de sano realismo.

Todas estas referencias históricas y actuales nos permitirían afirmar que, en contra del tópico que considera a la defensa y la política exterior como las "políticas de Estado" más típicas y caracterizadas, la regla es la discrepancia en estos terrenos, mientras que la excepción es la coincidencia y consenso en las mismas de las diferentes fuerzas políticas. Otra cosa bien diferente es que la estabilidad, fortaleza, capacidad de acción y prestigio del sistema político se ven reforzadas cuando se logra conformar una política de Estado, basada en un amplio consenso en estos terrenos tan sensibles. En síntesis, es deseable una "política de Estado" en estas cuestiones pero muy a menudo resulta imposible de alcanzar.

\section{Politica de Estado y politica del Estado: El papel del Parlamento en la conformación DE LA POLÍTICA DE DEFENSA}

Lo que sucede en la práctica habitual es que, en estas materias, la política del Gobierno se presenta habitualmente como política de Estado, sin que los partidos de oposición, en el caso de que no compartan los planteamientos oficiales, estén en condiciones de empeñarse a fondo en una resistencia a los mismos. Agotadas las vías parlamentarias de debate y discusión, que normalmente terminan con el triunfo de las posiciones gubernamentales, cada Gobierno hace de su política una política del Estado, aunque ésta no merezca la etiqueta de política de Estado, en sentido estricto. Sobre todo en su proyección exterior, la "cara" del Estado no es otra que la del Gobierno y en las relaciones internacionales no cuenta sino lo que dice y hace el Gobierno, con independencia del mayor o menor consenso en que se base. Desde fuera no hay más política de cada Estado que la política del Gobierno de turno.

En efecto, estas políticas, en cuanto inspiradoras de las relaciones internacionales, se proyectan en gran parte hacia el exterior y, lógicamente, se presentan como las "políticas del Estado" ya que lo son, sin ninguna duda; cualquiera que sea el debate interno sobre la acción exterior y cualesquiera que sean los resultados y las conclusiones del mismo, al final el Gobierno de turno hace de su política la política "del" Estado, aunque no sea una política "de" Estado en el sentido de haber sido apoyada y consensuada por los partidos políticos más significativos. Siempre hay una política "del" Estado -la del Gobierno- aunque ésta no sea una política "de" Estado, con independencia de cuáles hayan sido los mecanismos utilizados para la toma de las decisiones en esa materia.

Se puede argumentar que, en ciertas materias específicas del ámbito de la defensa, como sería el caso típico de las misiones en el exterior, cada vez de mayor actualidad, se puede conseguir una política de Estado si la decisión se somete al voto del Parlamento donde, por definición, se sientan todos los sectores significativos de la opinión nacional. Pero esto exige muchas y complejas matizaciones. En la inmensa mayoría de los países del mundo occidental democrático, la política de defensa es una de las competencias exclusivas del Poder Ejecutivo, sometida, claro está, al control del Legislativo, pero sin que éste, en principio, participe en la decisión, salvo en el caso específico de la declaración de guerra, institución esta última obsoleta en este momento histórico, como señalan la mayor parte de los constitucionalistas y de los internacionalistas ${ }^{8}$. En la Unión Europea, hasta ahora sólo Dinamarca y Alemania, por razones nacionales muy peculiares (en el caso alemán, como reacción al militarismo que llevó al país a las dos guerras mundiales) introducen en su ordenamiento jurídico preceptos que impiden la actuación exterior de sus Fuerzas Armadas sin previa autorización del Parlamento9.

Hay un caso específico, muy reciente y significativo al respecto. Nos referimos a los Paises Bajos, donde tras los bombardeos de la OTAN contra Serbia por la cuestión de Kosovo, en 1999, se abrió un debate nacional acerca del papel del Parlamento en relación con las misiones exteriores de las Fuerzas Armadas. Como consecuencia del mismo se modificó el artículo 100 de la Constitución, que quedó redactado de la siguiente forma: "Previamente al compromiso de uso o puesta a disposición de las Fuerzas Armadas para el mantenimiento o promoción del Estado de Derecho internacional, es preciso informar al Parlamento sobre la acción. Esta obligación incluye la provisión de información relativa en los casos de asistencia humanitaria en caso de conflicto armado". El apartado 2 del mismo 
artículo precisa: "La anterior obligación no se aplicará si se dan circunstancias perentorias, si bien se hará efectiva tan pronto como sea posible"10.

En España se había seguido hasta ahora el criterio de que las misiones exteriores de las Fuerzas Armadas -en cuanto parte componente de la política de defensa- eran competencia exclusiva del Gobierno en virtud del artículo 97 de la Constitución, según el cual "el Gobierno dirige la política interior y exterior, la Administración civil y militar y la defensa del Estado". No obstante, y aunque no existía ninguna norma que estableciese la obligación de informar al Parlamento de estas misiones, ha sido práctica habitual que los diferentes Gobiernos hayan informado a las Cámaras, principalmente y sobre todo al Congreso de los Diputados, del desarrollo de las misiones en el exterior de las Fuerzas Armadas. Estas sesiones informativas, celebradas con frecuencia en sesiones conjuntas de las Comisiones de Asuntos Exteriores y de Defensa del Congreso, unas veces lo han sido a petición e iniciativa del Gobierno, pero también, y no con menos frecuencia, a solicitud de los Grupos Parlamentarios. El mecanismo combinaba así la competencia del Gobierno en la política del defensa con la función de control de la acción del Gobierno que la misma Constitución (artículo 66) atribuye a las Cortes Generales.

Este sistema tradicional se ha visto alterado totalmente con la nueva Ley Orgánica de la Defensa Nacional que asigna al Congreso de los Diputados la competencia de "autorizar, con carácter previo la participación de las Fuerzas Armadas en misiones fuera del territorio nacional" (artículo 4.2). El artículo 17.1 reitera esta obligación ("Para ordenar operaciones en el exterior que no estén directamente relacionadas con la defensa de España o del interés nacional, el Gobierno realizará una consulta previa y recabará la autorización del Congreso de los Diputados"), pero en los apartados 2 y 3 del mismo artículo se matiza y flexibiliza esta obligación hasta dejarla, en la práctica, en pura fórmula de estilo con escasa vigencia efectiva ${ }^{11}$. En nuestra opinión, este precepto que atribuye al Congreso de los Diputados la facultad de autorizar, con carácter previo, la participación de las Fuerzas Armadas en misiones fuera del territorio nacional, entra, además, en contradicción con el artículo siguiente, el 5, de la nueva ley orgánica que, al fijar las competencias del Gobierno en este ámbito de la defensa atribuye al Ejecutivo la facultad de "acordar la participación de las Fuerzas Armadas en misiones fuera del territorio nacional".
La intervención obligada del Parlamento en estas cuestiones introduce un inconveniente elemento de rigidez en la acción del Gobierno, que muy frecuentemente tiene que tomar decisiones y asumir compromisos en breves períodos de tiempo y sin excesivos condicionamientos o caveats. Ésa fue la razón por la que en los Países Bajos mantuvieron la plena competencia del Gobierno a la hora de decidir la participación de sus Fuerzas Armadas en misiones en el exterior, con la obligación de la información previa al Parlamento. En el caso español, la rigidez e inconveniencia del precepto que exige la previa autorización parlamentaria se ha constatado, apenas iniciada la vigencia de la nueva Ley Orgánica de Defensa Nacional, con la polémica en torno a la participación -sin previa autorización parlamentaria y sin que se hubiera informado al Congreso de tal misión- de la fragata Álvaro de Bazán en el "grupo de combate" comandado por el portaaviones norteamericano Theodore Rooselvet, en misión en el Golfo Pérsico en el marco de la operación denominada "Libertad para Iraq".

Que la preceptiva aprobación por parte del Congreso de los Diputados se ha quedado convertida en una nueva fórmula de estilo, quedó bien a la vista con la participación española en UNIFIL, la operación de la ONU en el Líbano. El Congreso de los Diputados "aprobó" efectivamente esa misión, con amplísimo consenso, en septiembre de 2006. Pero cuando lo hizo el Gobierno ya tenía pactados y acordados con la ONU y con los otros paises participantes en la misión el tamaño y naturaleza del contingente español, el lugar donde había de desplegarse y las reglas de enfrentamiento que regirian la actuación del contingente. En suma, el Congreso de los Diputados sólo pudo decir "amén" a una misión ya diseñada y delimitada por el Gobierno, en uso en sus competencias.

Desde el punto de vista de la técnica legislativa, los preceptos citados de la nueva Ley Orgánica de Defensa Nacional son de una patente tosquedad y sólo pueden entenderse como concesión demagógica a los sectores más radicales que se opusieron a la intervención española en Iraq, decidida por el anterior Gobierno del Partido Popular. En aquel ambiente de asfixiante demagogia se "vendió" a la opinión pública que el Gobierno había metido a España en una guerra, cuando lo evidente es que aquel Gobierno nunca envió unidades de combate y se limitó, inicialmente, a enviar una misión humanitaria, centrada en un buque- 
hospital, para, más tarde, y cuando aparentemente habian terminado las operaciones militares, enviar una misión militar con funciones de reconstrucción nacional de Iraq, tras la dictadura y la guerra. En aquel conflicto, en suma, España no fue neutral porque apoyaba el derrocamiento del dictador Sadam Husein, pero tampoco fue beligerante, aunque así lo hiciera creer la entonces oposición socialista. Y puesto que estamos refiriéndonos al papel del Parlamento en la conformación de la política de defensa habria que recordar que, aunque el Gobierno del presidente Aznar no recabó la autorización del Congreso para esa misión exterior -como tampoco lo hizo el Gobierno del presidente González para la intervención militar en las operaciones de la primera Guerra del Golfo (1991)- porque ninguna norma establecía esa obligación, sí debe constatarse que el Congreso tuvo permanente información del desarrollo de la misión y que incluso una votación secreta, celebrada a instancias de la oposición, ratificó y apoyó la política que estaba llevando a cabo el Gobierno. Que el Gobierno del Partido Popular contara en aquel momento con mayoría absoluta en el Congreso, no empece en absoluto a la corrección de la votación desde el punto de vista de la técnica parlamentaria y de su legitimidad. Otra cosa es la valoración política acerca de su oportunidad o pertinencia que se quiera hacer de esa decisión.

La participación del Parlamento a la hora de decidir una misión exterior, ¿transforma, sin más, una "política de Gobierno" en "política de Estado"? La cuestión es muy discutible porque la solución depende no sólo del resultado de la votación y lo decisivo, a estos efectos, no es cuántos votos sino cuáles y de quiénes. Como "cámaras de registro" que son hoy día los Parlamentos, según afirman tantos constitucionalistas, el debate y la votación no añaden nada -o muy poco- a la decisión tomada por el Gobierno que, se supone, cuenta con una cómoda mayoría en la cámara parlamentaria. Por chocante que pueda parecer desde el punto de vista de la ortodoxia parlamentaria clásica, lo cierto es que una votación parlamentaria no transforma sin más a la "política del Gobierno" en una "política de Estado".

En todo caso, la participación del Parlamento en la toma de decisiones -que no en el control- en estas materias plantea más problemas de los que, seguramente, resuelve. La larga tradición que atribuye estas materias al Poder Ejecutivo no es caprichosa sino que se basa en sólidos fundamentos. La tradición constitucionalista occidental es muy constante al respecto. Como escribe un constitucionalista americano actual, John Yoo, "tanto la teoría política, en cuanto desarrollada principalmente por pensadores como Locke, Montesquieu y Blackstone como la historia constitucional angloamericana desde el siglo XVII hasta el tiempo en que se redactó la Constitución de los Estados Unidos, establece que los asuntos exteriores eran provincia de la rama ejecutiva de gobierno". Y no hay duda al respecto de que lo que se dice de la política exterior se puede aplicar también al ámbito de la defensa ${ }^{12}$.

El pensamiento de los Padres Fundadores de los Estados Unidos -e inspiradores de su Constitución- no deja dudas al respecto. Madison, ocupándose específicamente de la defensa, en algunos de los capítulos de The Federalist, escribió aquello de que "la guerra es la madre del Ejecutivo", con lo que quería decir que en situación de conflicto es indispensable la dirección del Ejecutivo. Algo parecido escribió Hamilton en otro de los capítulos de la misma clásica obra: "De todas las responsabilidades y obligaciones de un gobierno, la dirección de la guerra exige, del modo más peculiar, aquellas cualidades que distinguen el ejercicio del poder por una sola mano". Y más adelante: "La dirección de la guerra implica la dirección de la fuerza común y el poder de dirigir y emplear la fuerza común forma parte esencial y usual de la definición de la autoridad ejecutiva".

Se podría decir que, en todo caso, la declaración de guerra que es, en este terreno, la más importante decisión imaginable es una competencia exclusiva de los Parlamentos. Pero incluso eso es dudoso. Como ya hemos señalado más arriba, en la presente situación internacional la declaración de guerra es una práctica obsoleta porque la guerra de agresión está prohibida por la legalidad internacional y tanto la guerra defensiva como otro tipo de conflictos, como los de imposición o mantenimiento de la paz u otros similares, por su propia naturaleza y porque se suelen iniciar en el ámbito y a instancias de Naciones Unidas o de otras organizaciones internacionales, como la OTAN, no exigen tampoco ese trámite. En el caso de los Estados Unidos, el mismo John Yoo ha señalado que en 207 años de vigencia de su Constitución, aquel país ha comprometido tropas en el exterior en 125 ocasiones. Sin embargo, sólo en cinco de las mismas se produjo una declaración de guerra ${ }^{13}$. 


\section{Política de DEFENSA Y OPINIÓN PÚBLICA}

Aunque formalmente el Parlamento representa a la soberanía nacional y es, en consecuencia, la única representación legitima de la opinión pública, no cabe duda de que ésta a veces se manifiesta en desacuerdo con las posiciones adoptadas mayoritariamente por el Parlamento, a través de los medios de comunicación, de encuestas de opinión y de movilizaciones. El peso y la influencia de la opinión pública, así entendida, en las cuestiones de política exterior y de defensa es cada vez mayor, aunque al tiempo, se da la paradoja del escaso interés e información que tiene la opinión pública sobre estas cuestiones. Lo que podemos llamar "divorcio" entre Parlamento y opinión pública no es un tema nuevo, sobre todo en relación con las intervenciones militares en el exterior y nos ocuparemos de ello un poco más adelante. Vayamos ahora a la cuestión del escaso interés de la opinión pública sobre estas cuestiones.

Hay que tener en cuenta, efectivamente, que la opinión pública, salvo casos excepcionales, no sigue con demasiada atención los asuntos relacionados con la política exterior y la defensa. Las encuestas son reiteradamente contundentes al respecto. El común de los ciudadanos ni tiene una información detallada por estas cuestiones ni sitúa a la política exterior y de defensa entre sus prioridades, según muestran una y otra vez los barómetros de opinión. En esta situación, los partidos no suelen suscitar estas cuestiones en sus actuaciones públicas, más allá de algunas frases retóricas. Basta ver la escasa atención, que, proporcionalmente, se presta a estos temas en las campañas electorales, tanto en los programas de partido como en los mítines y otros actos del mismo carácter. Los partidos, en respuesta a lo que se supone que son predisposiciones de la opinión pública, tienen una tendencia manifiesta a abordar todos estos asuntos en "clave interior". Es bien sabido, por ejemplo, que en las campañas para las elecciones al Parlamento Europeo (en la medida en que se considere que la política respecto la Unión Europea es una cuestión exterior) apenas si se abordan los temas institucionales europeos o las políticas comunes, como sería el caso de la Política Exterior y de Defensa (PESO) y, dentro de ella, de la Política Europea de Seguridad y Defensa (PESD).

El único tema relacionado con la defensa que suscitó pasiones en España en la primera etapa de la democracia ha sido el de la OTAN. Como escribe el profesor Díez Nicolás:
"La decisión de Calvo Sotelo de realizar la incorporación de España a la OTAN sobre la base del acuerdo parlamentario, pero sin el respaldo de una consulta popular, aunque legalmente correcta, fue políticamente incorrecta, o al menos inoportuna. En efecto, desde el día siguiente a su aprobación parlamentaria el $\mathrm{PSOE}$, como principal partido de la oposición, dispuso de una bandera electoral que habría de darle mucho juego. El eslogan electoral 'OTAN, de entrada NO', se convirtió en el principal reclamo para las elecciones de 1982, pues llevó a votar, y por tanto a votar por el PSOE, a todos los grupos y grupúsculos políticos de izquierda que, en otras circunstancias, posiblemente no habrian votado, pero que encontraron una razón para hacerlo y demostrar así su pacifismo, su rechazo de los gastos de armamento, su rechazo a la energía nuclear, su antiamericanismo, su antibelicismo, etc. La opinión pública, en solos unos meses de movilización por parte del PSOE, del PCE y de otros partidos de izquierda, había pasado de ser mayoritariamente favorable a la incorporación de España a la OTAN, a ser mayoritariamente contraria a ella, como demostraron todas las encuestas realizadas en aquellas fechas sobre esta cuestión"14.

La OTAN ha sido, pues, un tema divisivo $y$, de hecho, lo sigue siendo, aunque de una manera mucho más matizada. Según el Informe INCIPE 2003 "los españoles hemos ido modificando los enormes recelos que suscitaba entre nosotros la Alianza Atlántica. En 2002, el $63 \%$ de los españoles consultados apuesta por nuestra permanencia en su seno, incrementando en más de 10 puntos los resultados de 1997". En relación con otro tema que ha provocado históricamente "opiniones encontradas", el de las bases norteamericanas en suelo español, el mismo Informe señala que "sus partidarios y detractores han moderado sus opiniones", aunque en relación con el conflicto de Iraq (2003), "a juicio del 74\% de los españoles, nuestro país no debería permitir a las Fuerzas Armadas norteamericanas la utilización de sus bases en territorio español para el ataque a Iraq" Otra cuestión relacionada con la defensa que se aborda en el Informe es de la profesionalización de las Fuerzas Armadas, que cuenta con el apoyo mayoritario, aunque si bien "una mayoria relativa de los españoles aprecia que el número de soldados que integran nuestras Fuerzas Armadas es suficiente... uno de cada tres españoles consideran que el paso a un ejército profesional ha reducido los efectivos necesarios para nuestras necesidades de la defensa"15. 
Un caso especifico de ese "divorcio" entre opinión pública e instancias oficiales, más frecuente y más antiguo de lo que puede parecer a primera vista, es de las misiones militares en el exterior, decididas por el Gobierno de turno en el uso de sus competencias constitucionales, y apoyadas incluso por el Parlamento, pero que se enfrentan a un rechazo más o menos amplio, pero constatable de la opinión pública. Se trata de un viejo problema que ya fue planteado en la década de los ochenta del siglo XIX en un interesante y largo estudio Public Opinión and Lord Beaconsfield (1875-1880) publicado en 1886 y cuyo autor es George Carslake Thompson. Se trata de un análisis, desde el punto de vista de la opinión pública, de la llamada "cuestión de Oriente", que enfrentaba entonces a los imperios zarista y otomano y que llevaría al Congreso de Berlín (1884-1885). Mientras la opinión pública británica mantenía una postura pro rusa y veía con satisfacción el debilitamiento de los turcos, opresores de los cristianos ortodoxos que habitaban en los países balcánicos, la política oficial del Gobierno Disraeli (Lord Beaconsfield) era favorable a Turquía, tanto en aplicación de la tradición británica de la balance of power que, a la búsqueda del equilibrio se inclinaba siempre por la parte más débil del cualquier conflicto, como porque la rivalidad ruso-británica en Asia central (lo que se llamó "el Gran Juego") y la cada vez más preocupante proximidad rusa a los dominios británicos en la India, dictaban a Whitehall el mantenimiento de la tradicional política de apoyo a Turquía, que había incluso Ilevado a la Guerra de Crimea (1854-1856). Ya en el siglo XX un autor clásico en los estudios de opinión pública, Paul F. Lazarsfeld, volvió sobre la obra de Carslake Thompson y se preguntaba hasta qué punto puede un Gobierno resistir a las presiones de la opinión pública "cuando existen signos inequívocos de que la población en su conjunto no está de acuerdo con su política"16.

A partir del problema ruso-turco Thompson hace unas interesantes reflexiones sobre la opinión pública que siguen teniendo validez. Estima que es un error considerarla como "una e indivisible", insistiendo que sobre un tema concreto "a menudo surge la controversia". "Debe recordarse -escribe- que 'opinión pública', 'la voluntad de la nación' y otras frases del mismo tipo no son realmente sino metáforas por medio de las cuales el pensamiento y la voluntad son atribuidos a una mente singular, mientras que el 'público' y la 'nación' son agregados de muchas gentes". Más adelante escribe que "en tanto que la opinión pública está organizada de alguna forma, como el Parlamento, la opinión pre- ponderante puede, por supuesto, ser fácilmente conocida... pero hay ocasiones en se siente que la verdadera opinión del país es algo distinto de la del Parlamento. ¿Cómo va a ser reconocida esta verdadera opinión?"

Seguramente en la actualidad se contestaría a la pregunta de Thomson diciendo que para eso están las encuestas de opinión. Pero inmediatamente surgen una larga serie de preguntas de no fácil contestación: ¿Deben predominar en todo caso las encuestas sobre la voluntad del Parlamento? ¿Tienen los gobiernos que practicar la llamada encuestocracia, esto es utilizar los sondeos como único criterio para sus decisiones politicas? En la actualidad es obvio que cualquier gobierno no puede dejar de hacer un seguimiento permanente de la opinión pública pero, si es muy lógico no perder de vista las diferentes corrientes de opinión, no lo parece tanto dar a las encuestas un valor prescriptivo. Las encuestas pueden condicionar pero no determinar la política del gobierno en el ámbito de la defensa y en cualquier otro y queda a la prudencia política el grado de influencia que deben tener en la toma de decisiones. Sobre todo teniendo en cuenta que una de las características de la opinión pública, tal y como aparece medida por las encuestas, en su versatilidad.

En el caso español es de especial interés la evolución de la opinión pública en relación con la participación española de conflictos u operaciones en el exterior. Esta cuestión ha sido especialmente estudiada por Díez Nícolás que, en el libro citado, ha analizado especialmente esa evolución en relación la guerra en el Golfo Pérsico. Escribe que aquel conflicto sirvió para poner nuevamente de manifiesto los deseos pacifistas más o menos permanentes de la sociedad española de no intervenir en conflictos fuera de nuestras fronteras... y de cierta necesidad de marcar diferencias respecto a los Estados Unidos y, posiblemente -añade- a cierta constante en la sociedad española consistente en tomar partido por el más débil. Lo curioso, sin embargo, es que si de septiembre de 1990 a febrero de 1991 la opinión pública fue mayoritariamente opuesta a la participación española -a pesar de que el Congreso por amplísima mayoría y con el apoyo del primer partido de la oposición había votado a favor de la participación española, "en marzo... una vez concluidas las actuaciones bélicas y aceptada la tregua, los españoles tomaron conciencia de haber formado parte de los ejércitos aliados vencedores en esa contienda, algo que no sucedía en España desde las guerras napoleónicas"17. 
No voy a entrar en el análisis de la crisis de Iraq -a la que sí he aludido de pasada más arriba- que, por una parte, refleja las mismas pautas de pacifismo a ultranza por parte de amplios sectores de la opinión pública pero, por la otra, pone en evidencia una utilización demagógica de esa situación por parte de la oposición, algo que no se dio en la anterior Guerra del Golfo.

\section{Conclusiones}

1. A todos los Estados les interesa, especialmente en sus relaciones internacionales, mantener la continuidad de sus grandes opciones políticas, como expresión de conjuntos nacionales sólidos, coherentes y sin fisuras. La "política de Estado" asi entendida consolida su legitimidad y prestigio y garantiza ante los otros sujetos internacionales la permanencia y continuidad de sus compromisos.

2. Conseguir una "política de Estado" obliga a los gobiernos a negociar con los grupos políticos más significativos y representativos hasta alcanzar posiciones que puedan ser apoyadas en el más amplio consenso posible. Alcanzar ese consenso no supone "hacer pasar por el aro" a los demás sino escuchar sus puntos de vista hasta alcanzar puntos de acuerdo apoyados mayoritariamente.

3. Aunque es deseable alcanzar "políticas de Estado" en ámbitos tan estratégicos como la política exterior y la defensa, la historia y la experiencia política muestran que con mucha frecuencia no se alcanza el necesario consenso y la "política del Gobierno" pasa como "política del Estado".

4. Una política de Estado, apoyada por los principales partidos políticos, es más fácil que sea aceptada por los sectores mayoritarios de la opinión pública, aunque nunca es seguro.

5. Una ideal política de Estado en defensa, mantenida en el tiempo y resistente a las alternancias políticas, sería el instrumento más adecuado para lograr una cultura de defensa que, en la actualidad, es casi inexistente en nuestro país.

\section{NOTAS}

1 Vid. una discusión al respecto en Joseph S. Nye Jr.: The Paradox of American Power, Oxford University Press, 2002. Especialmente oportuno el capitulo 5 "Redefining the nacional interest", pp. 137 y ss.

2 Vid. Florentino Portero: "Buenismo y alianza de civilizaciones", en Valentí Puig (coordinador): El fraude del buenismo, FAES, 2005.

3 Walter Russell Mead: Special Providence, Routledge, 2002.

4 Henry Kissinger: Diplomacy, Simon \& Schuster, 1994.

5 Pierre Hassner Justin Vaïse: Washington et le monde. Dilemmes d'une superpuissance, Eds. Autrement, 2003.

Recibido: 22 de enero de 2008 Aceptado: 27 de marzo de 2008
6 Madeleine Albright: Memorias, Planeta, 2003, pp. 346-347.
7 Vid. Maurice Vaïsse: La grandeur. Politique étrangère du general de Gaulle 1958-1969, Fayard, 1998.

8 Vid. Elisa Pérez Vera y Alejandro J. Rodríguez Carrión: "Artículo 63. Atribuciones Internacionales del Rey", en Comentarios a la Constitución Española de 1978 (dirigidos por Óscar Alzaga Villamil), tomo $\mathrm{V}$ (artículos 56 a 65), Cortes Generales, Editoriales de Derecho Reunidas, 1997, especialmente pp. 274-278. Vid. también José Luis Fernández-Flores y de Funes: El Derecho de los Conflictos Armados, Ministerio de Defensa, 2001, pp. 369-376.

9 Vid. Los nuevos retos y la reforma institucional de las Naciones Unidas, trabajos de la Ponencia creada en el Congreso de los Diputados, Cortes Generales, 1995. Vid. especialmente 
el apartado 19 "El papel del Parlamento" y el cuadro 2 "Competencias del Parlamento en misiones bajo mandato de Naciones Unidas" donde se hace un análisis comparativo de las competencias de los Parlamentos de los principales países de la UE y de la OTAN, pp. 27-31.

10 Vid. Lorenzo Cotino Hueso: El modelo constitucional de Fuerzas Armadas, Madrid, 2002, pp. 320 y nota 206.

11 Dicen así estos apartados del citado artículo 17 de la Ley Orgánica de la Defensa Nacional: "17.2. En las misiones en el exterior que, de acuerdo con compromisos internacionales, requieran una respuesta rápida 0 inmediata a determinadas situacio- nes, los trámites de consulta previa y autorización se realizarán mediante procedimientos de urgencia que permitan cumplir con dichos compromisos. 17.3. En los supuestos previstos en el apartado anterior, cuando por razones de máxima urgencia no fuera posible realizar la consulta previa, el Gobierno someterá al Congreso de los Diputados lo antes posible la decisión que haya adoptado para la ratificación, en su caso".

12 Vid. John Yoo: The Powers of War and Peace (The Constitution and Foreign Affairs after 9/11), The University of Chicago Press, 2005, p. 19.

13 Ibidem, p. 12. Las cinco ocasiones en las que los Estados Unidos han declarado formalmente la guerra según
Yoo han sido las siguientes: La guerra de 1812 contra Gran Bretaña; la guerra contra México de 1848; la guerra hispano-norteamericana de 1898 y las dos guerras mundiales.

14 Juan Díez Nicolás: Identidad nacional y cultura de defensa, Ed. Síntesis, 1999, p. 185.

15 Salustiano del Campo y Juan Manuel Camacho: Laopinión pública españolay la política exterior.InformeINCIPE2003, INCIPE y Real Instituto Elcano, 2003, p. 17.

16 Vid. Paul F. Lazarsfeld: "Public opinión and the classical tradition," en Robert0. Carlson(editor):CommunicationandPublic Opinion, Praeger, 1975, p. 627.

17 J. Díez Nicolás: ob. cit., pp. 226 y 232-233. 